\title{
Most common concurrent infections with COVID-19
}

\author{
Ahmed R. Zein ${ }^{1 *}$, Emad A. Almuqati², Hebah H. Taher ${ }^{3}$, Sarah S. Jahlan ${ }^{4}$, \\ Fatemah H. AlKhamis ${ }^{3}$, Abdulwahab A. Almansour ${ }^{5}$, Shoroq F. Alhazmi ${ }^{6}$, Maha M. Alshehri ${ }^{7}$, \\ Mohamed I. Obaidat ${ }^{8}$, Muath A. Alshehri ${ }^{9}$, Zahra H. Alhoori ${ }^{10}$
}

\author{
${ }^{1}$ Department of Intensive Care Unit, King Fahad General Hospital, Jeddah, Saudi Arabia \\ ${ }^{2}$ Department of Intensive Care Unit, Al Noor Specialist Hospital, Mecca, Saudi Arabia \\ ${ }^{3}$ College of Medicine, Arabian Gulf University, Manama, Bahrain \\ ${ }^{4}$ Department of Pediatrics, King Khalid Hospital, Tabuk, Saudi Arabia \\ ${ }^{5}$ College of Medicine, King Saud University, Riyadh, Saudi Arabia \\ ${ }^{6}$ College of Medicine, Northern Border University, Arar, Saudi Arabia \\ ${ }^{7}$ College of Medicine, King Khalid University, Abha, Saudi Arabia \\ ${ }^{8}$ Department of General Medicine/Family Medicine, Astar Clinics, Manama, Bahrain \\ ${ }^{9}$ Department of Emergency Medicine, Almuzahmiyah General Hospital, Riyadh, Saudi Arabia \\ ${ }^{10}$ Department of Internal Medicine, Salmaniya Medical Complex, Manama, Bahrain
}

Received: 03 January 2022

Accepted: 18 January 2022

*Correspondence:

Dr. Ahmed R. Zein,

E-mail: ahmadragab@gmail.com

Copyright: (c) the author(s), publisher and licensee Medip Academy. This is an open-access article distributed under the terms of the Creative Commons Attribution Non-Commercial License, which permits unrestricted non-commercial use, distribution, and reproduction in any medium, provided the original work is properly cited.

\begin{abstract}
Concurrent infections are a common complication of viral respiratory infections. They pose diagnostic challenges due to an overlap of similar symptoms, resistance to treatment and extending length of hospital stay. In this review we will extensively discuss the most common concurrent infections in patients with coronavirus disease 2019 (COVID-19). A thorough literature search was conducted in online databases such as PubMed, Google Scholar and included systematic reviews, meta-analyses, prospective and retrospective cohort studies in this review. Bacterial co-infections are the most common concurrent infections in patients with COVID-19 succeeded by viral and fungal co-infections. The prevalence of co-infections in COVID-19 patients is higher in intensive care units (ICU). Gram negative bacteria such as Klebsiella pneumoniae, Pseudomonas aeruginosa and Escherichia coli, whereas gram positive bacteria such as Staphylococcus aureus are common pathogens for bacterial co-infections. The findings for the most common viral co-infection are inconsistent however, larger number of studies report respiratory viral co-infections such as influenza and respiratory syncytial virus. Fungal co-infections in COVID-19 patients are most commonly caused by the Candida spp. and occur predominantly in patients admitted in the ICU and are associated with high mortality and morbidity in COVID-19 patients. Continuous research on concurrent infections occurring in COVID-19 is essential. Larger prospective studies based on stratified groups of age, gender, and both ICU and non-ICU settings should be conducted. Studies on microbial susceptibility can lend more weight to empirical antibiotic and antifungal therapy. Early diagnosis of concurrent infections in COVID-19 is imperative to prevent poor patient outcomes.
\end{abstract}

Keywords: COVID-19, Concurrent, Co-infection, Bacterial, Viral, Fungal

\section{INTRODUCTION}

Coronaviruses are single stranded positive ribonucleic acid (RNA) viruses belonging to the Coronoviridae family. In December 2019, an unfamiliar coronavirus was identified during an outbreak causing a respiratory illness in Wuhan, China. ${ }^{1}$ It was officially named as the severe acute respiratory syndrome coronavirus-2 (SARS-CoV-2) whereas the disease caused by SARS-CoV-2 was called coronavirus disease 2019 (COVID-19). ${ }^{2}$ Due to the high 
transmissibility of the virus, COVID-19 rapidly progressed as a pandemic infecting millions of people globally, leading to high in-hospital admissions, overburdening health care systems, causing more than 5 million deaths worldwide and major losses in business and productivity. ${ }^{2,3}$

The United States Centers for Disease Control and Prevention (CDC) describe a concurrent infection as being infected with two or more separate causative agents simultaneously causing an overlap of symptoms, therefore creating challenges in reaching a definitive diagnosis. ${ }^{4,5}$ The term co-infection is also used throughout literature when a concurring infection occurs with the primary infection on initial presentation. Since the emergence of the COVID-19 pandemic, the role of co-infections in COVID-19 is still unclear. ${ }^{6}$ All concurrent infections can occur in two forms. A community acquired infection (CAI), when a co-existing infection is diagnosed at the time of initial infection or within 48 hours of hospital admission, and a hospital acquired infection (HAI) occurs when a co-existing infection is not present at the time of initial infection but diagnosed 48 hours after hospital admission. HAI are also known as secondary infections or super-infections. $^{7}$ Studies have reported a $45 \%$ coinfection and rate of secondary infections in patients with COVID-19. ${ }^{8}$

Concurrent infections are a common occurrence in viral respiratory infections. Co-infections pose diagnostic challenges in identification due to similarities in symptoms, difficulties in management and treatment, causing an extended length of hospital stay, and even escalation of treatment to the intensive care unit (ICU) with a need for mechanical ventilation. ${ }^{9}$ Concurrent infections often have a poor prognosis, increased morbidity and mortality especially in high-risk populations such as the immunocompromised, patients with chronic comorbidities and elderly patients. ${ }^{4}$ The purpose of this review is to discuss some of the most common concurrent infections occurring in patients diagnosed with COVID19.

\section{METHODS}

An extensive research was conducted from electronic databases such as PubMed, Medline Embase, Google Scholar and Cochrane Library. To avoid missing potential studies, a further manual search for papers was done through Google Scholar. Previous studies including case reports, literature reviews, and cohort studies have been included. Only studies in English were included.

\section{DISCUSSION}

A large systematic review with 72 studies and a metaanalysis with 68 studies was conducted in Saudi Arabia. The combined studies had a total of 31,953 SARS-CoV-2 infected patients. The overall pooled proportions of SARSCoV-2 patients with bacterial co-infections were $15.9 \%$
(95\% confidence interval 13.6-18.2). The rate for viral coinfections was $6.6 \%$ whereas, fungal co-infections rates were $3.7 \%$. In bacterial co-infected SARS-CoV-2 patients, an analysis between two subgroups of both ICU, non-ICU patients and only-ICU patients was done. The rate of bacterial co-infection was higher in the only-ICU patients' group with a rate of $22.2 \%$ (95\% CI 16.1 to 28.4) whereas the rate of co-infection in both ICU and non-ICU group was $14.8 \%$. Similarly, an analysis for fungal co-infected SARS-CoV-2 patients between the two sub-groups showed a significant difference. The rate of fungal coinfection in the only-ICU group was higher 9.6\% (95\% CI 6.8 to 12.4 ) as compared to the both ICU and non-ICU patients which was $2.7 \%$. However, in the respiratory viral co-infected SARS-CoV-2 patients, an analysis between both subgroups showed an identical proportion between both ICU, non-ICU patients' $(6.6 \%)$ and the only-ICU patients' $(6.6 \%)$. Bacterial co-pathogens were reported in 49 out of 72 studies (68\%), accounting for $57.3 \%$ of reported co-infections. Respiratory viral co-pathogens were reported in 44 out of 72 studies $(61.1 \%)$, accounting for $39.5 \%$ of the reported co-infections, while fungal copathogens were reported in 16 out of 72 studies $(22.2 \%)$ accounting for only $3.2 \%$ of all reported co-infections. ${ }^{10}$

Similar findings were observed in a systematic review and meta-analysis on co-infections in patients hospitalized with COVID-19 conducted by Lansbury et al. Out of 30 studies, 3834 patients were included. The overall pooled proportion of co-infections in hospitalized COVID-19 patients was $12 \%$. Studies were further segregated into categories of ICU only patients and a mixed population of patients admitted both in the ward and ICU. The rate of bacterial co-infection was $7 \%$ (95\% CI 3 to 12$)$. The subgroup analysis in bacterial co-infections showed a significantly higher rate in ICU only patients with $14 \%$ (95\% CI 5 to 26), as compared to a $4 \%$ in mixed hospitalized patients. A pooled analysis showed an estimated rate of $3 \%$ for viral co-infections, however, there was no statistically significant difference in the sub-group analysis in viral co-infections with a rate of $5 \%$ in the ICU only group as compared to $3 \%$ viral co-infection in the mixed patient population. Fungal co-infections with 4 pathogens were identified in only 3 studies out of 30 studies. ${ }^{11}$ Similarly, a retrospective cohort study in Spain revealed out of 989 patients, 72 patients had 88 had coinfections, from which 74 were bacterial co-infections, 7 were viral and 7 were fungal co-infections. ${ }^{12}$

The difference in prevalence between bacterial and viral co-infections can also be observed in a study on coinfections among COVID-19 patients conducted in the United States. In this study, the authors Singh et al compared the rate of co-infections in SARS-CoV-2 positives to SARS-CoV-2 negatives. Out of 50,419 respiratory samples of nasopharyngeal, oropharyngeal and sputum, 4259 samples were positive with SARS-CoV-2. The rate of bacterial co-infection was $33.17 \%$ in SARSCoV-2 positive patients, whereas the rate of viral coinfection was $3.42 \%$. Although the rates of bacterial and 
viral co-infection in SARS-CoV-2 positive patients were lower in comparison to SARS-CoV-2 negative patients (35.45 \% and $8.46 \%$ ), bacterial co-infection still showed predominance in comparison to viral co-infections in SARS-CoV-2 positive patients. ${ }^{8}$ However, a few studies have reported conflicting results. Fattorini et al discuss 13 studies with 733 patients. Viral co-infections were identified in $17.2 \%$ patients (126/733) bacterial coinfections were found in $11.8 \%$ patients $(86 / 733)$, whereas fungal co-infections were found in only $1.8 \%$ patients (13/733). ${ }^{13}$ Similarly, a systematic review by Musuuza et al reported out of 118 studies, 67 had co-infections (57\%). The estimated pooled prevalence rate of viral co-infections was $10 \%$ (95\% CI: $6 \%-14 \%)$ while viral superinfections were $4 \%$. The rate of bacterial coinfections was $8 \%$ and bacterial superinfections was $20 \%$ (95\% CI: $13 \%-28 \%$ ) whereas the rate of fungal co-infections was $4 \%$, and fungal superinfections, $8 \%$. A high prevalence of coinfections was observed in the non-ICU population at $29 \%$ (95\% CI: $14 \%-46 \%$ ), 18\% among combined patients of ICU and non-ICU patients, whereas ICU only patients had a co-infection rate of $16 \% .{ }^{14}$

\section{Concurrent bacterial infections}

Concurrent infections with bacterial pathogens are known to be the leading cause of mortality in pandemics such as influenza and viral pneumonias. ${ }^{8,15}$ A retrospective-cohort observational study on the microbiological profile of hospitalized COVID-19 patients was conducted in United Arab Emirates (UAE) from February to July 2020. The results of the study reported 392 laboratory-confirmed coinfections from 29,802 COVID-19 patients. In bacterial co-infections, the most common pathogens identified were gram-negative organisms such as Pseudomonas aeruginosa, Klebsiella pneumoniae, and Escherichia coli, Stenotrophomonas maltophilia, and Acinetobacter baumannii. The common gram-positive organisms were Staphylococcus epidermidis, Enterococcus faecalis and Staphylococcus aureus and methicillin resistant Staphylococcus aureus (MRSA). ${ }^{15}$ A similar retrospective study on bacterial coinfections among COVID-19 positive patients was conducted in Bahrain. A total of 1380 COVID-19 positive patients were included in the study of which 261 had a confirmed bacterial co-infection (19\%). Gram negative bacteria was isolated from $34.7 \%$ patients, of which the most common pathogens were Klebsiella pneumoniae, Pseudomonas aeruginosa, MDR Acinetobacter baumannii, Escherichia coli, Stenotrophomonas maltophilia (S. maltophilia), and Enterobacter cloacae (E. cloacae). Gram positive bacteria were also isolated from $34.7 \%$ patients, and the most common pathogens were Staphylococcus hominis ( $S$. hominis), Staphylococcus epidermidis, Enterococcus faecium, Enterococcus faecalis, and Staphylococcus aureus. Hospital acquired Infections accounted for $70 \%$ of which $75 \%$ were due to gram negative bacteria and $11.8 \%$ were due to gram positive bacteria. ${ }^{7}$ According to the retrospective cohort study in Spain, 74 bacterial coinfections were identified in 61 out of 88 co-infected patients positive with COVID-19. Furthermore, in 25 patients, 30 community acquired bacterial co-infections were reported $(2.5 \%)$ and were mostly due to gram positive bacteria (S. pneumoniae, aureus, MRSA). Similarly, 44 hospital acquired bacterial co-infections or superinfections were diagnosed in 38 patients $(3.8 \%)$ and were mostly due to Gram-negative bacteria ( $P$. aeruginosa, E. coli, and $K$. pneumoniae). ${ }^{12}$ In the study by Singh et al, the infection rate of bacterial pathogens was lower in SARS-CoV-2 positive patients in comparison with SARS-CoV-2 negative patients, except, $S$. aureus which was the only bacterial pathogen that was reportedly higher in SARSCoV-2 positives at a rate of $13.17 \%$, as compared to SARS-CoV-2 negatives $11.64 \%{ }^{8}$

\section{Concurrent viral infections}

Various studies present conflicting results on common concurrent viral infections. According to the systematic review conducted in Saudi Arabia, from $61.1 \%$ studies, the most common respiratory viruses were Epstein Barr virus (EBV), human herpes virus 6 (HHV6), influenza A virus, human metapneumovirus and adenovirus. ${ }^{10}$ Similarly, according to Singh et al, the most prevalent viral coinfection among SARS-CoV-2 positive cases was the EBV. ${ }^{8}$ Higher EBV infections have also been reported among COVID-19 patients in Wuhan, China. EBV exists in a latent form after a primary infection and gets reactivated during periods of immunosuppression. Therefore, EBV infections can undergo reactivation during a COVID-19 infection as a result of the host's immune mediated response. ${ }^{8,16}$ Furthermore, EBV deoxyribonucleic acid (DNA) is found in $95.2 \%$ of COVID-19 patients admitted in the medical ICU, and $83.6 \%$ of patients admitted in the surgical ICU. ${ }^{16}$ On the contrary, according to the meta-analysis by Lansbury et al, viral co-infections were identified in 14 studies from which the most common viral co-infection was due to respiratory syncytial virus (RSV) making $16.8 \%$ of detections, followed by influenza A virus $(15.5 \%$ detections $){ }^{11}$ Similar findings were observed in the retrospective study in Spain, where community acquired viral co-infections were detected in 7 out of 989 patients (0.6\%). Influenza $A$ was most common followed by $R S V$ and herpetic virus. ${ }^{12}$ In contrast to the above findings, according to a systematic review in Iran, out of 33 studies, 10484 patients with COVID-19 were identified. The pooled prevalence of viral coinfections was estimated to be $12.58 \%$. The most common type of viruses among all viral co-infections were blood borne viruses (pooled prevalence: $12.48 \%$; 95\% CI: 8.57 to 16.93 ) whereas respiratory viruses were the least common (pooled prevalence: $4.32 \%$ ). Some of the common blood borne viruses are human immunodeficiency virus (HIV) and hepatitis $\mathrm{C}$ virus $(\mathrm{HCV}){ }^{16}$

\section{Concurrent fungal infections}

Fungal co-infections are more common in COVID-19 patients admitted in the ICU. ${ }^{10}$ They are a major cause of 
mortality in mechanically ventilated COVID-19 patients. ${ }^{17}$ A report in South Africa discusses fungal co-infections are common in COVID-19 patients. The most common fungal co-infections are caused by Aspergillus fumigatus, Aspergillus flavus and Candida albicans, Candida tropicans. ${ }^{18}$. Patients admitted with COVID-19 pneumonia may develop acute respiratory distress syndrome (ARDS) and become highly susceptible to invasive aspergillosis caused by Aspergillus spp leading to COVID-19 associated aspergillosis (CAPA), for which cases have been reported worldwide. ${ }^{19-21}$ A narrative review reported a cohort of 43 patients, which identified 3 patients with candidemia. The common pathogens causing fungal co-infections were Candida albicans, Candida tropicalis and Candida parapsilosis. Furthermore, a retrospective study by Huges et al reported 3 fungal coinfections caused by Candida albicans in a cohort of 836 patients admitted with COVID-19. In addition to Aspergillus and Candida spp, fungal co-infections with Pneumocystis jirovecii and mycoses such as Saccharomyces have been reported in COVID-19 patients. ${ }^{19,21}$

\section{Concurrent infections with tuberculosis}

COVID-19 and pulmonary tuberculosis (PTB) has been reported as a co-infection in various studies. ${ }^{19}$ A literature review discusses the co-infection of TB and COVID-19. Only 8 studies with a total of 80 patients were found. PTB causes Mycobacterium Tuberculosis in majority of the patients. COVID-19 and a TB co-infection was subdivided into groups based on different clinical scenarios including, TB diagnosed before COVID-19, TB diagnosed after COVID-19 and diagnosis of COVID-19 in patients receiving treatment for TB, were classified as COVID-19 with active TB. From 80 patients, 70 patients were reported as having active pulmonary TB during a diagnosis of COVID-19. A TB-COVID-19 infection was identified among both genders but predominantly males. ${ }^{22}$ Diseases due to human immunodeficiency virus (HIV) and PTB lead to severe immunodeficiency and therefore, pose a higher risk of co-infections. TB is the leading cause of death in patients with HIV. The concurrence of TB, HIV and COVID-19 poses a major public health challenge, especially in endemic countries. Patients with COVID-19 increase the risk of $\mathrm{TB}$ and thus, makes diseased individuals highly susceptible to the causal nexus of HIVTB-COVID-19. ${ }^{23}$ A systematic review on different studies with COVID-19 and HIV/TB or only TB was conducted. A total of 21 studies were included in the systematic review, with 28,387 COVID-19 patients. Among them, 1294 were a co-infection of COVID-19 TB, whereas 1094 were COVID-19/HIV/TB. Occurrence of TB was also subgrouped into previous TB and current TB. The previous TB group included patients where sputum was negative for acid fast bacilli and the diagnosis for TB was based on exposure to $\mathrm{TB}$ in the past, symptoms and radiograph images indicating active disease, whereas current TB was sputum smears positive for acid fast bacilli. According to the meta-analysis, current TB showed a strong risk of
COVID-19 in HIV-infected cases OR 2.01 (95\% CI 1.103.66), compared to uninfected HIV cases OR 1.30. TB occurrence pooled results between subgroup COVID19/HIV/TB and COVID-19/TB was OR 1.67 (95\% CI 1.06-2.65). The test for subgroup differences was not statistically significant. Therefore, the meta-analysis on the occurrence of TB among both groups of, COVID19/HIV/TB and COVID-19/TB demonstrated that COVID-19 had a higher risk of occurring in current TB subgroup. ${ }^{23}$

Although sufficient literature is available on concurrent infections, but results of co-infections and super infections are often discussed cumulatively, thereby limiting correct interpretation. Many systematic reviews discussed occurrence of co-infections in SARS-CoV-2 in combination with other coronaviruses such as SARS-CoV and Middle East respiratory syndrome coronavirus (MERS-CoV), again limiting true interpretation of results on SARS-CoV-2 co-infections only. There is limited literature on the occurrence of COVID-19 as a co-infection with TB and HIV in and a lack of comparison with other microbial pathogens. Furthermore, studies on occurrence and prevalence of common concurrent infections are limited, as majority of the studies have different outcomes of interest such as clinical features, length of hospital stay, prognosis, status of mechanical ventilation, mortality and susceptibility to antibiotics. It is strongly recommended to discuss rates of co-infections and super infections in COVID-19 separately for a more precise understanding on occurrence and prevalence of microbial infections. Prospective studies on various microbiological pathogens causing co-infections in COVID-19 among various demographic variables such as age, gender, associated chronic co-morbidities and different hospital settings of wards and ICU, including subgroups of patients with and without mechanical ventilation should also be considered.

\section{CONCLUSION}

The most common concurrent infection in patients with COVID-19 are bacterial co-infections followed by viral whereas fungal co-infection are the least common. Bacterial co-infections are caused predominantly by gramnegative organisms; however, they are only surpassed by Staphylococcus aureus which is the most common gram positive organism that causes bacterial co-infections. The most common viral co-infections are due to respiratory viruses such as influenza and respiratory syncytial virus. Fungal co-infections are most commonly found in COVID-19 patients admitted in the ICU. They are usually hospital acquired infections or superinfections and associated with increased mortality and morbidity. More research needs to be conducted on COVID-19 and concurrent infections with TB and HIV. Studies on microbial antibiotic susceptibility can steer antimicrobial stewardship programs and prove highly beneficial for improved outcomes in patients with COVID-19 and concurrent infections. A strong focus and clinical awareness should be placed on early diagnosis and 
treatment of concurrent infection in COVID-19 to prevent adverse sequence of events.

Funding: No funding sources

Conflict of interest: None declared

Ethical approval: Not required

\section{REFERENCES}

1. Li Y, Wang H, Wang F, Lu X, Du H, Xu J, Han F, Zhang L, Zhang M. Co-infections of SARS-CoV-2 with multiple common respiratory pathogens in infected children: A retrospective study. Medicine (Baltimore). 2021;100(11):e24315.

2. Shereen MA, Khan S, Kazmi A, Bashir N, Siddique R. COVID-19 infection: Origin, transmission, and characteristics of human coronaviruses. J Adv Res. 2020;24:91.

3. Khurana S, Singh P, Sharad N, Kiro VV, Rastogi N, Lathwal A, Malhotra R, Trikha A, Mathur P. Profile of co-infections \& secondary infections in COVID19 patients at a dedicated COVID-19 facility of a tertiary care Indian hospital: Implication on antimicrobial resistance. Indian J Med Microbiol. 2021;39(2):147-53.

4. Kwon WJ, Li G, Zheng M, Kaur H, Magbual N, Dalai S. Superinfections and coinfections in COVID-19separating the signal from the noise. Medpage Today April. 2020;28.

5. Mushtaq MB, Qadri MI, Rashid A. Concurrent infection with dengue and malaria: an unusual presentation. Case Rep Med. 2013.

6. Petersen FC, Dahle UR, Nicolau B, Casals-Pascual C. COVID-19: Looking Into the Overlooked. Front Mol Biosc. 2020;7.

7. Saeed NK, Al-Khawaja S, Alsalman J, Almusawi S, Albalooshi NA, Al-Biltagi M. Bacterial co-infection in patients with SARS-CoV-2 in the Kingdom of Bahrain. World J Virol. 2021;10(4):168.

8. Singh V, Upadhyay P, Reddy J, Granger J. SARSCoV-2 respiratory co-infections: Incidence of viral and bacterial co-pathogens. Int $\mathrm{J}$ Infect Dis. 2021;105:617-20.

9. Cox MJ, Loman N, Bogaert D, O'Grady J. Coinfections: potentially lethal and unexplored in COVID-19. The Lancet Microbe. 2020;1(1):e11.

10. Alhumaid S, Al Mutair A, Al Alawi Z. Coinfections with bacteria, fungi, and respiratory viruses in patients with SARS-CoV-2: a systematic review and meta-analysis. Pathogens. 2021;10(7):809.

11. Lansbury L, Lim B, Baskaran V, Lim WS. Coinfections in people with COVID-19: a systematic review and meta-analysis. J Infect. 2020;81(2):26675 .

12. Garcia-Vidal C, Sanjuan G, Moreno-García E. Incidence of co-infections and superinfections in hospitalized patients with COVID-19: a retrospective cohort study. Clin Microb Infect. 2021;27(1):83-8.
13. Fattorini L, Creti R, Palma C, Pantosti A. Bacterial coinfections in COVID-19: an underestimated adversary. Annali dell'Istituto superiore di sanita. 2020;56(3):359-64.

14. Musuuza JS, Watson L, Parmasad V, PutmanBuehler N, Christensen L, Safdar N. Prevalence and outcomes of co-infection and superinfection with SARS-CoV-2 and other pathogens: A systematic review and meta-analysis. PloS one. 2021;16(5):e0251170.

15. Senok A, Alfaresi M, Khansaheb H, Nassar R, Hachim M, Al Suwaidi H, et al. Coinfections in Patients Hospitalized with COVID-19: A Descriptive Study from the United Arab Emirates. Infect Drug Resist. 2021;14:2289-96.

16. Malekifar P, Pakzad R, Shahbahrami R, Zandi M, Jafarpour A, Rezayat SA, et al. Viral Coinfection among COVID-19 Patient Groups: An Update Systematic Review and Meta-Analysis. Biomed Res Int. 2021;2021:5313832.

17. Zhou F, Yu T, Du R, Fan G, Liu Y, Liu Z, et al. Clinical course and risk factors for mortality of adult inpatients with COVID-19 in Wuhan, China: a retrospective cohort study. Lancet. 2020;395(10229):1054-62.

18. Ezeokoli O, Pohl C. Opportunistic pathogenic fungal co-infections are prevalent in critically ill COVID-19 patients: Are they risk factors for disease severity? South Afr Med J. 2020;110(11):1081-5.

19. Abdoli A, Falahi S, Kenarkoohi A. COVID-19associated opportunistic infections: a snapshot on the current reports. Clin Exp Med. 2021:1-20.

20. Nasir N, Farooqi J, Mahmood SF, Jabeen K. COVID19-associated pulmonary aspergillosis (CAPA) in patients admitted with severe COVID-19 pneumonia: An observational study from Pakistan. Mycoses. 2020;63(8):766-70.

21. Pemán J, Ruiz-Gaitán A, García-Vidal C. Fungal coinfection in COVID-19 patients: Should we be concerned? Revista iberoamericana de micologia. 2020;37(2):41-6.

22. Mishra AK, George AA, Sahu KK, Lal A, Abraham G. Review of clinical profile, risk factors, and outcomein patients with Tuberculosis and COVID19. Acta Bio Medica: Atenei Parmensis. 2021;92(1).

23. Tamuzi JL, Ayele BT, Shumba CS. Implications of COVID-19 in high burden countries for HIV/TB: A systematic review of evidence. BMC Infect Dis. 2020;20(1):1-18.

Cite this article as: Zein AR, Almuqati EA, Taher HH, Jahlan SS, AlKhamis FH, Almansour AA, et al. Most common concurrent infections with COVID-19. Int J Community Med Public Health 2022;9:1108-12. 